\title{
Les professionnels de la santé buccodentaire du Canada et la gestion des antimicrobiens
}

\author{
Bureau du dentiste en chef du Canada ${ }^{1}$
}

\section{Résumé}

La résistance aux antimicrobiens est une préoccupation mondiale, car elle présente une grave menace à notre capacité de traiter les maladies infectieuses. Le Canada a pris des mesures pour relever le défi de la résistance aux antimicrobiens depuis 1997. Ces mesures comprennent une stratégie nationale à quatre piliers : surveillance, gestion, prévention et contrôle des infections, recherche et innovation. Les dentistes jouent un rôle important en contribuant aux efforts consacrés à ces quatre piliers, en particulier celui de la gestion. Des études démontrent que les ordonnances d'antibiotiques pour des raisons de santé buccodentaire augmentent avec le temps et que $60 \%$ à $80 \%$ des antibiotiques prescrits en milieu dentaire ne sont pas nécessairement indiqués sur le plan clinique. L'élaboration, la promotion et la mise en œuvre d'initiatives visant à promouvoir l'utilisation optimale des antimicrobiens partout au Canada nécessiteront la collaboration de nombreux intervenants, y compris la collectivité de la santé buccodentaire. La résistance aux antimicrobiens et la gestion des antimicrobiens font déjà l'objet de discussions au sein de la profession dentaire au Canada; cependant, il reste encore du travail à faire dans divers domaines, notamment l'utilisation par les dentistes des lignes directrices courantes fondées sur des données probantes et des protocoles d'ordonnance mis en œuvre par leurs instances dirigeantes pour garantir une ordonnance appropriée d'antibiotiques au besoin et un accès abordable et opportun aux services de santé buccodentaire par les Canadiens.
Cette oeuvre est mise à la disposition selon les termes de la licence internationale Creative Commons Attribution 4.0

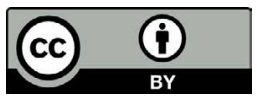

Affiliation

1 Bureau du dentiste en chef du Canada, Agence de la santé publique du Canada, Ottawa, ON

Correspondance :

claudia.gorenko@canada.ca
Citation proposée : Bureau du dentiste en chef du Canada. Les professionnels de la santé buccodentaire au Canada et la gestion des antimicrobiens. Relevé des maladies transmissibles au Canada 2020;46(11/12):423-6. https://doi.org/10.14745/ccdr.v46i1112a02f

Mots-clés : résistance aux antimicrobiens, gestion, antibiotiques, ordonnance, meilleures pratiques, dentistes

\section{Introduction}

La découverte et le développement des antibiotiques font partie des plus grandes réalisations médicales du $X X^{e}$ siècle; cependant, avec une utilisation accrue, les bactéries peuvent développer une résistance aux antibiotiques au fil du temps. L'Organisation mondiale de la Santé (OMS) a fait de la résistance aux antimicrobiens une priorité. L'OMS appelle à une action coordonnée au niveau mondial pour réduire l'émergence et la propagation de la résistance aux antimicrobiens et pour que chaque pays se dote d'un plan d'action national (1). Le Canada a pris des mesures pour relever les défis de la résistance aux antimicrobiens depuis 1997 et ces mesures comprennent une stratégie nationale à quatre piliers : surveillance, gestion, prévention et contrôle des infections, recherche et innovation (2-4). Comme le souligne le rapport Pleins feux de I'administratrice en chef de la santé publique du Canada 2019, le Canada a fait des progrès et les taux de résistance aux antimicrobiens sont inférieurs à ceux de nombreux autres pays du monde (5). Cependant, il reste encore du travail à faire.
En tant que fournisseurs de soins de santé et prescripteurs, les dentistes ont un rôle important à jouer en contribuant aux efforts consacrés à la stratégie nationale à quatre piliers, en particulier la gestion. La gestion des antimicrobiens renvoie à des interventions coordonnées conçues pour promouvoir, améliorer, surveiller et évaluer une utilisation judicieuse et appropriée des antimicrobiens afin de préserver leur efficacité future et de favoriser et de protéger la santé humaine et animale (2). Les sections suivantes examineront de plus près les habitudes d'ordonnance dans la pratique de la santé buccodentaire par rapport à celles des médecins praticiens ainsi que les initiatives de la résistance aux antimicrobiens déjà en cours, et donneront un aperçu des travaux de la gestion des antimicrobiens à venir dans la médecine dentaire canadienne. 


\section{Utilisation des antimicrobiens et pratiques d'ordonnance}

Au Canada, environ $92 \%$ des antibiotiques sont utilisés en dehors du milieu hospitalier de soins de courte durée; $89 \%$ sont prescrits par des médecins, $8 \%$ par des dentistes et $3 \%$ par du personnel infirmier, des pharmaciens et des optométristes (2). En médecine dentaire, les antibiotiques sont utilisés à deux fins : prophylactique et thérapeutique. Les antibiotiques prophylactiques sont utilisés pour prévenir l'infection et ce type d'ordonnance est plus répandu que les antibiotiques thérapeutiques, lesquels sont utilisés pour traiter une infection existante (5). L'Association dentaire canadienne appuie les lignes directrices de l'American Heart Association concernant la prophylaxie antibiotique avant certaines procédures de santé buccodentaire visant à prévenir l'endocardite infectieuse. Ces lignes directrices stipulent qu'en raison d'un nombre croissant de données probantes, les risques liés à la prise d'antibiotiques préventifs l'emportent sur les avantages pour la plupart des patients (6). Malgré ces lignes directrices, dans une étude de cohorte rétrospective menée aux États-Unis examinant des données de 2011 à 2015, on a découvert que les dentistes aux États-Unis prescrivaient souvent une prophylaxie primaire aux patients en bonne santé subissant des procédures de santé buccodentaire invasives même si les preuves à l'appui d'une telle utilisation sont minimes et peu concluantes (7). Plus précisément, les chercheurs ont constaté que plus de $80 \%$ des antibiotiques prescrits pour la prophylaxie des infections avant la visite chez le dentiste n'étaient pas nécessaires (7).

Les études au Royaume-Uni et aux États-Unis démontrent qu'entre $60 \%$ et $80 \%$ des antibiotiques prescrits en milieu dentaire ne sont pas nécessairement indiqués sur le plan clinique (7-9). Malheureusement, à I'heure actuelle, les données publiées sur les pratiques d'ordonnance d'antibiotiques des dentistes au Canada sont limitées. Les données canadiennes disponibles montrent une tendance à la baisse du taux d'ordonnance d'antimicrobiens chez les médecins (10). Une augmentation des ordonnances chez les dentistes (2010 à 2012) a été suivie d'un taux généralement stable de 2012 à 2015 (10). Une étude en Colombie-Britannique a également montré que l'utilisation globale d'antibiotiques et les ordonnances prescrites par les médecins ont diminué de 18,2 \% entre 1996 et 2013 (11). Cependant, on a signalé une augmentation de $62,2 \%$ des ordonnances d'antibiotiques prescrites par les dentistes en Colombie-Britannique au cours de la même période, au moment où I'utilisation des recommandations et des lignes directrices aurait dû entraîner une baisse (11).

L'Association dentaire canadienne a tenté de documenter les pratiques d'ordonnance des dentistes au Canada en 2017. Les résultats d'un sondage en ligne indiquent que la plupart des dentistes au Canada déclarent prescrire des antibiotiques selon les meilleures données probantes et directives cliniques à leur disposition (12). Les résultats montrent également qu'il existe des préoccupations, notamment la surutilisation de certains types d'antibiotiques, des disparités concernant les états de santé et les procédures dentaires nécessitant une prophylaxie antibiotique pour la prévention de l'endocardite infectieuse et les affections dentaires nécessitant des antibiotiques thérapeutiques. Une autre préoccupation est un manque apparent de sensibilisation chez certains dentistes aux changements dans les directives d'ordonnance d'antibiotiques (12). Alors que le sondage était basé sur des informations autodéclarées et que la taille de l'échantillon était petite ( $n=1035$, ce qui représente un taux de réponse de $16,5 \%$ ), les résultats pointent vers le besoin de recherches supplémentaires pour mieux comprendre les pratiques d'ordonnance des dentistes canadiens.

De nombreux facteurs, au-delà des preuves et des pratiques exemplaires, contribuent à la décision de prescrire un antibiotique. Ces facteurs peuvent inclure les recommandations d'autres professionnels de la santé, les attentes des patients, les lignes directrices peu claires, obsolètes ou changeantes ou le manque de connaissance des lignes directrices récentes, I'incertitude du diagnostic et les contraintes de temps (5). Selon une étude réalisée par Suda et al. (7), les raisons de l'augmentation des taux d'ordonnance d'antibiotiques chez les dentistes sont l'utilisation croissante d'implants dentaires, une population vieillissante, la sous-assurance qui propulse les antibiotiques comme un substitut à la chirurgie buccale, la lenteur de l'adoption des nouvelles lignes directrices, le manque de sensibilisation au rôle des dentistes dans la résistance aux antimicrobiens et la pression du médecin et du patient. Ces caractéristiques sont semblables à celles associées à la prescription excessive d'antibiotiques par les médecins (7). En outre, une revue exploratoire a révélé que les raisons de prescrire des antibiotiques thérapeutiques comprennent un temps limité pour les rendez-vous d'urgence, les contraintes de temps et les patients non assurés qui n'ont pas les moyens de se payer un traitement approprié (13). Néanmoins, à l'instar des autres professionnels de santé prescripteurs, le défi pour les dentistes est de s'assurer qu'ils ne prescrivent des antibiotiques que lorsque c'est nécessaire et en stricte conformité avec la posologie et la durée recommandées pour cet antibiotique (en visant le temps le plus court possible pour l'effet thérapeutique recherché).

Contribuant aux problèmes liés à la résistance aux antimicrobiens, certains patients consultent des médecins dans les services d'urgence des hôpitaux. Entre 2001 et 2010, les visites aux services d'urgence des États-Unis par des personnes âgées de 20 à 29 ans représentaient $42 \%$ de toutes les visites pour les maux de dents au service d'urgence, se classant au cinquième rang des raisons les plus courantes pour toute visite au service d'urgence et au troisième rang des visites au service d'urgence dans ce groupe d'âge (14,15). En 2019, un autre groupe de chercheurs a mené un examen de la portée à l'aide de données canadiennes et américaines pour cartographier les facteurs préliminaires associés à l'utilisation des services d'urgence pour de problèmes dentaires non traumatiques (15). 
Bien que les chercheurs aient indiqué plusieurs limites, leurs résultats préliminaires montrent que les patients visitent les services d'urgence en raison des facteurs démographiques, d'accessibilité, d'influences économiques et sociales avec le revenu et l'incapacité de payer des soins comme facteurs les plus courants.

\section{Initiatives de gestion dentaire sur la résistance aux antimicrobiens au Canada}

Certaines initiatives de gestion sont déjà en cours pour sensibiliser et éduquer les professionnels dentaires sur la résistance aux antimicrobiens. Par exemple, la résistance aux antimicrobien et la gestion des antimicrobiens font déjà l'objet de discussions au sein de la profession dentaire au Canada. L'Association dentaire canadienne sensibilise à la résistance aux antimicrobiens, encourage la mobilisation des professionnels canadiens de la santé buccodentaire et publie des articles sur la résistance aux antimicrobiens dans la revue de l'Association dentaire canadienne (16). L'Association dentaire canadienne a également participé aux collaborations nationales parrainées par l'Agence de la santé publique du Canada (l'Agence). Au sein de l'Agence, le Bureau du dentiste en chef du Canada travaille également avec l'équipe de la résistance aux antimicrobiens de l'Agence et avec les principaux intervenants nationaux en santé buccodentaire pour permettre à la profession de s'aligner sur les meilleures pratiques d'ordonnance afin d'atténuer les risques de la résistance aux antimicrobiens. L'Association canadienne des dentistes en milieu hospitalier participera à la Table ronde d'action SoinsSantéCAN pour élaborer un plan d'action national de gestion des antimicrobiens et collabore avec les dentistes et les médecins spécialistes des maladies infectieuses pour communiquer l'importance cruciale d'une utilisation responsable des antimicrobiens (17).

Sur le plan des ressources pour les cliniciens, aux États-Unis, le service de la santé publique du Massachusetts a mis au point une trousse d'outils pour la gestion des antibiotiques pour les cliniciens en santé buccodentaire, qui se compose de deux courtes vidéos YouTube en plus de webinaires (18). Au Canada, I'Agence a contribué au parrainage de modules de formation sur la résistance aux antimicrobiens, donnant aux dentistes canadiens l'accès à une formation continue ciblée grâce aux modules de formation en ligne de l'Université de Waterloo (19). Les dentistes peuvent également guider leurs patients vers de I'information facile à lire sur la résistance aux antimicrobiens dans les sites Web « Des pilules contre tous les microbes » (20) et «Choisir avec soin Canada/Antibiotiques » (21).

À l'avenir, différentes approches devraient être envisagées par la profession dentaire pour lutter contre la résistance aux antimicrobien. L'élaboration, la promotion et la mise en œuvre d'initiatives visant à promouvoir l'utilisation optimale des antimicrobiens partout au Canada nécessiteront la collaboration de nombreux intervenants (22). Les gouvernements devraient examiner davantage les disparités d'accès aux soins de santé buccodentaire et les inégalités auxquelles sont confrontés des segments de la population afin d'éviter des consultations inutiles chez les médecins et d'autres fournisseurs de soins pour des problèmes de santé buccodentaire $(14,15)$. Cela réduirait l'ordonnance inutile d'antibiotiques comme il est susmentionné, par exemple dans les services d'urgence (14). Les gouvernements fédéral, provinciaux et territoriaux et leurs organismes de réglementation respectifs jouent également un rôle clé dans la responsabilité partagée de la surveillance et de l'évaluation des lignes directrices, une composante essentielle des stratégies de la gestion des antimicrobiens (22). L'OMS et les Centers for Disease Control and Prevention (CDC) des États-Unis formulent des politiques sur la gestion des antimicrobiens (23). La responsabilité, la formation, le suivi et l'établissement des rapports sur les modèles d'ordonnance et l'éducation des cliniciens et des patients sont des exemples des composantes essentielles des CDC de la gestion des antimicrobiens en clinique ambulatoire (23). Les gouvernements, les associations dentaires et les organismes de réglementation peuvent s'appuyer sur ces éléments comme point de départ.

Les initiatives de la gestion des antimicrobiens sont soutenues au niveau du gouvernement et des associations dentaires $(2,6,22)$. Les possibilités de formation continue, les lignes directrices professionnelles et la sensibilisation devraient être utilisées et promues parmi les dentistes en plus des recherches supplémentaires au Canada pour mieux comprendre les habitudes d'ordonnance d'antibiotiques des cliniciens dentaires.

\section{Intérêts concurrents}

Aucun.

\section{Financement}

Ce travail est soutenu par l'Agence de la santé publique du Canada.

\section{Références}

1. Organisation mondiale de la Santé. Résistance aux antimicrobiens. Genève (Suisse) : OMS; juillet 2020. https://www.who.int/fr/news-room/fact-sheets/detail/ antimicrobial-resistance

2. Agence de la santé publique du Canada. Lutter contre la résistance aux antimicrobiens et optimiser leur utilisation : un cadre d'action pancanadien. Élement 3: Intendance. Ottawa (ON) : ASPC; 2017. https://www.canada.ca/fr/sante-canada/ services/publications/medicaments-et-produits-sante/ lutter-contre-resistance-antimicrobiens-optimiser -utilisation-cadre-action-pancanadien.html\#a3.3 
3. Agence de la santé publique du Canada. Évaluation de la coordination des activités associées à la résistance aux antimicrobiens (RAM) à l'Agence de la santé publique du Canada. Ottawa (ON) : BAE/ASPC; 2019. https://www. canada.ca/fr/sante-publique/organisation/transparence/ rapports-gestion/evaluation/sommaire-evaluatio n-horizontale-initiative-federale-lutte-contre-vihsida-2013-2014-2017-2018/rapport-evaluatio $\mathrm{n}$-coordination-activites-associees-resistance-antimicrobiens. html

4. National Collaborating Centre for Infectious Diseases. Surveillance of Antimicrobial Resistance and Antimicrobial Utilization in Canada. NCCID; 2014. https://nccid.ca/publications/surveillance-of-antimicrobia l-resistance-and-antimicrobial-utilization-in-canada/

5. Agence de la santé publique du Canada. Manipuler avec soin : préserver les antibiotiques aujourd hui et demain. Pleins feux de l'administratrice en chef de la santé publique du Canada 2019. Ottawa (ON) : ASPC, 2019 (accédé 2020-06-12). https://www.canada.ca/content/dam/ phac-aspc/documents/corporate/publications/chief-publi c-health-officer-reports-state-public-health-canada/ preserving-antibiotics/Final_CPHO_Report_EN_June6_2019. pdf

6. Oberoi SS, Dhingra C, Sharma G, Sardana D. Antibiotics in dental practice: how justified are we. Int Dent J 2015;65(1):4-10. DOI PubMed

7. Association dentaire canadienne. La prévention de l'endocardite infectieuse. ADC; 2014 (accédé 2020-09-02). https://www.cda-adc.ca/fr/about/position_statements/ infectiveendocarditis/

8. Suda KJ, Calip GS, Zhou J, Rowan S, Gross AE, Hershow RC, Perez RI, McGregor JC, Evans CT. Assessment of the appropriateness of antibiotic prescriptions for infection prophylaxis before dental procedures, 2011-2015. JAMA Netw Open 2019;2(5):e193909. DOI PubMed

9. Patrick A, Kandiah T. Resistance to change: how much longer will our antibiotics work? Fac Dent J. 2018;9(3):104-11. DO।

10. Thompson W, Tonkin-Crine S, Pavitt SH, McEachan RR, Douglas GV, Aggarwal VR, Sandoe JA. Factors associated with antibiotic prescribing for adults with acute conditions: an umbrella review across primary care and a systematic review focusing on primary dental care. J Antimicrob Chemother 2019;74(8):2139-52. DOl PubMed

11. Agence de la santé publique du Canada. Système canadien de surveillance de la résistance aux antimicrobiens Rapport de 2017. Ottawa, 2017 (accédé 2020-09-01). http://www.canada.ca/en/public-health/services/ publications/drugs-health-products/canadian-antimicrobia I-resistance-surveillance-system-2017-report-executivesummary.html
12. Marra F, George D, Chong M, Sutherland S, Patrick DM. Antibiotic prescribing by dentists has increased: why? J Am Dent Assoc 2016;147(5):320-7. DOI PubMed

13. Association dentaire canadienne. Prescription d'antibiotiques. Mode de pratique des dentistes au Canada. ADC, 2018;5(1):24-7. https://www.cda-adc.ca/fr/services/ essentials/2018/issue1/24/

14. Stein K, Farmer J, Singhal S, Marra F, Sutherland S, Quiñonez $C$. The use and misuse of antibiotics in dentistry: $A$ scoping review. J Am Dent Assoc 2018;149(10):869-884.e5. DOl PubMed

15. Lewis CW, McKinney CM, Lee HH, Melbye ML, Rue TC. Visits to US emergency departments by 20- to 29-year-olds with toothache during 2001-2010. J Am Dent Assoc 2015;146(5):295-302.e2. DOI PubMed

16. Association dentaire canadienne. L'essentiel de l'ADC. Résistance antimicrobienne: Inverser la tendance. ADC; 2017. https://www.cda-adc.ca/fr/services/essentials/2017/ issue1/files/assets/common/downloads/publication.pdf

17. Canadian Association of Hospital Dentists. Antibiotic Stewardship. https://cahd-acdh.ca/antibiotic-stewardship/

18. Kennedy E, Klevens RM. Massachusetts Department of Public Health, Bureau of Infectious Disease and Laboratory Sciences. Antibiotic Stewardship Toolkit for Oral Health Clinicians. MDPHGIS; 2020. http://bit.ly/ DentalStewardshiplnformation

19. University of Waterloo. School of Pharmacy. Antimicrobial Stewardship in Primary Care Continuing Education Program (accédé 2020-08-10). https://uwaterloo.ca/ pharmacy/degrees-and-professional-development/ distance-education-continuing-professional-development/ antimicrobial-stewardship-primary-care-continuing-education

20. Alberta Health Services and the British Columbia Centre for Disease Control. Resources for Dentists and Dental Hygienists. AHS and BCCDC; 2019. http:// www.dobugsneeddrugs.org/health-care-professionals/ resources-dentists-dental-hygienists/

21. Antibioticswise.ca. Antibiotics and Dental Care. https://antibioticwise.ca/topics/antibiotics-and-dental-care/

22. Réseau pancanadien de santé publique, Partenaires en santé publique. Gestion des antimicrobiens. CRSP; 2016. http:// www.phn-rsp.ca/pubs/anstew-gestan/pdf/pub-fra.pdf

23. Teoh L, Thompson W, Suda K. Antimicrobial stewardship in dental practice. J Am Dent Assoc 2020 Aug;151(8):589-95. DOl PubMed 